\title{
artigo
}

Medeiros, R.R.P.; Costa, E.S.; Pereira da Silva, J.R.; Pereira da Silva, A.; Carvalho, L.F.F.; Sousa da Silva, G.N.;

Autocuidado materno no período puerperal: estudo de revisão da literatura

\section{Autocuidado materno no período puerperal: estudo de revisão da literatura}

\author{
Maternal self-care in the puerperal period: literature review study \\ El auto cuidado materno en el período puerperal: estudio de revisión de la literatura
}

\begin{abstract}
RESUMO
Objetivo: descrever a importância dos cuidados maternos no período puerperal encontradas na literatura e relatar a importância da enfermagem nos cuidados maternos durante o puerpério. Método: revisão integrativa da literatura, realizada no mês de março de 2020, nas bases de dados indexadas a Biblioteca Virtual em Saúde-BVS, que são: Literatura Latino-Americana e do Caribe em Ciências da Saúde- LILACS, SciELO - Scientific Electronic Library Online, Literatura Internacional em Ciências da Saúde- MEDLINE e Portal Periódicos Capes/MEC, através dos descritores, usando o operador AND. O corpus da revisão foi composto por 9 artigos que foram organizados e arquivados em pastas e construído cinco quadros. Resultados: as mulheres ainda têm pouco conhecimento a respeito do seu autocuidado no período puerperal e no cuidado com o recém-nascido. Conclusão: compreendeu-se a necessidade de existirem mais trabalhos sobre o autocuidado materno e incentivar os profissionais enfermeiros para a orientação específica para puérpera.
\end{abstract}

DESCRITORES: Puerpério; Cuidados; Enfermagem.

\section{ABSTRACT}

Objective: describing the importance of maternal care in the puerperium found in the literature and reporting the importance of nursing in maternal care during the puerperium. Method: integrative literature review, carried out in March 2020, on data bases indexed to the Virtual Library in Salud-VHL, which is: Latin American Literature and the Caribbean in Health Sciences- LILACS, SciELO - Scientific Electronic Library Online, International Literature in Health Sciences - MEDLINE and Portal Capi / MEC Journals, through descriptors, using the AND operator. The review corpus was composed of 9 articles that were organized and archived in carpets and built a five frames. Results: women have a lot of knowledge about their self-care in the puerperium and in the care of the newborn child. Conclusion: it is understood that it is necessary to work more on maternal self-care and encourage nursing professionals to provide specific guidance to women who have recently given birth.

DESCRIPTORS: Puerperium; Care; Nursing.

\section{RESUMEN}

Objetivo: describir la importancia del cuidado materno en el puerperio encontrada en la literatura y reportar la importancia de la enfermería en el cuidado materno durante el puerperio. Método: revisión integradora de la literatura, realizada en marzo de 2020, en las bases de datos indexadas a la Biblioteca Virtual en Salud-BVS, que son: Literatura Latinoamericana y del Caribe en Ciencias de la Salud- LILACS, SciELO - Biblioteca Electrónica Científica Online, Literatura Internacional en Ciencias de la Salud MEDLINE y Portal Periódicos Capes / MEC, a través de los descriptores, utilizando el operador AND. El corpus de revisión estuvo compuesto por 9 artículos que se organizaron y archivaron en carpetas y se construyó cinco cuadros. Resultados: las mujeres aún tienen poco conocimiento sobre su autocuidado en el puerperio y en el cuidado del recién nacido. Conclusión: se entendió que es necesario trabajar más en el autocuidado materno y alentar a los profesionales de enfermería a brindar orientación específica a las mujeres que han dado a luz recientemente.

DESCRIPTORES: Puerperio; Cuidado; Enfermería.

RECEBIDO EM: 19/10/2020 APROVADO EM: 10/11/2020

\section{Rosicleide Rúbia Pereira Medeiros}

Enfermeira. Pós-graduada em obstetrícia e neonatologia. Mestranda no programa de pós-graduação em educação, trabalho e inovação em medicina na Universidade Federal do Rio Grande do Norte.

ORCID: 0000-0002-3442-7999 


\section{Erlânia Souza Costa}

Enfermeira. Pós-graduada em obstetrícia e neonatologia.

ORCID: 0000-0002-5018-0404

\section{Janine Rosaline Pereira da Silva}

Enfermeira. Pós-graduada em obstetrícia e neonatologia.

ORCID: 0000-0002-2555-3299

\section{Aline Pereira da Silva}

Enfermeira. Pós-graduada em obstetrícia e neonatologia.

ORCID: 0000-0001-8185-9471

\section{Luciane de Fátima Fernandes de Carvalho}

Enfermeira. Pós-graduanda em obstetrícia e neonatologia.

ORCID: 0000-0002-2484-6129

\section{Glaydes Nely Sousa da Silva}

Enfermeira. Pós-graduada em Unidade de Terapia Intensiva. Mestre em Saúde da Família pela Faculdade de Enfermagem Nova Esperança.

ORCID: 0000-0002-6564-657X

\section{INTRODUÇÃO}

0 período puerperal é um momento de vivência singular para a mulher, trazendo consigo inúmeras transformações, desde as questões sociais, culturais, até as de conhecimento ${ }^{(1)}$. É uma fase de relevância no ciclo de vida da mulher, e diversas vezes é esquecida, além de ser dada muita ênfase ao recém-nascido, prejudicando os cuidados femininos no pós-parto ${ }^{(2)}$. Nesse período, a mulher é incentivada pelos familiares e simultaneamente por profissionais de saúde, a preocupar-se com o recém-nascido, e a maioria das vezes se restringe a vacinação e avaliação do bebê $\hat{e}^{(3)}$.

Durante o período puerperal ocorrem algumas modificações, que se direcionam ao psicológico, a anatomia da mulher e a sua própria fisiologia ${ }^{(4)}$. Além disso, existem as modificações psicológicas, pois esta mulher precisa de apoio em relação ao seu autocuidado e a sua nova rotina com o recém-nascido, deixando-a vulnerável ${ }^{(5)}$.

A importância dos cuidados maternos no período puerperal está relacionada a intercorrências que podem ocorrer nesse momento. A hemorragia puerperal é uma delas, que corresponde a $75 \%$ das complicações graves, e podem ocorrer no puerpério imediato ${ }^{(4)}$. Outra intercorrência que pode ocorrer é a infecção puerperal, que
A importância dos cuidados maternos no período puerperal está relacionada a intercorrências que podem ocorrer

nesse momento. A

hemorragia puerperal

é uma delas, que

corresponde a $75 \%$

das complicações

graves, e podem

ocorrer no

puerpério imediato. quando existe a ausência da assistência, a mulher pode sofrer complicações gravíssimas ${ }^{(3)}$. Além disso, o sistema psicológico também é afetado, e a mulher fica exposta a depressão pós-parto, e baby-blues, caso ela não tenha o apoio necessário para cuidar de si e do recém-nascido( ${ }^{(6)}$.

É importante que o enfermeiro, em suas consultas que antecedem o período puerperal, oriente essa mulher ao que vai acontecer ${ }^{(7)}$. Além disso, deve ser incentivado o autocuidado, desde a medidas simples até as ditas como complexas pelas mulheres, tais como: o banho, a higienização íntima, os cuidados com as mamas, as medicações, os contraceptivos, sexualidade no puerpério, dentre outros cuidados ${ }^{(8)}$. Com isso, estudos que possibilitem estimular a visualização da importância dos cuidados direcionados a genitora no período puerperal são relevantes. Este estudo tem como objetivo: descrever a importância do conhecimento sobre autocuidado materno no período puerperal e relatar a importância da enfermagem na assistência materna no puerpério.

\section{MÉTODO}

Trata-se de uma revisão integrativa da literatura, realizada no mês de março de 2020, nas bases de dados indexadas a 
Biblioteca Virtual em Saúde-BVS, que são: Literatura Latino-Americana e do Caribe em Ciências da Saúde- LILACS, SciELO - Scientific Electronic Library Online, Literatura Internacional em Ciências da Saúde- MEDLINE e Portal Periódicos Capes/MEC, através dos descritores "Puerpério" and "Cuidados" and "Enfermagem", utilizando o operador booleano AND. Para guiar o estudo, definiu-se como questão norteadora: qual a importância do conhecimento sobre autocuidado materno no período puerperal e da assistência de enfermagem no referido período, descritos em artigos científicos? Além disso, foram seguidos os passos conforme o PRISMA.

Foram encontrados e analisados 18 artigos relacionados com a temática. A partir da leitura, tiveram com critério de inclusão: textos publicados no período de 2009 a 2020, com a temática sobre cuidados maternos, puerpério, cuidados de enfermagem, pesquisas realizadas no Brasil e Exterior e textos em português, espanhol e inglês. Justifica-se o período estabelecido para busca das publicações, devido a necessidade de reunir o maior número de textos publicados relacionados à temática, buscando maior abrangência. Como critérios de exclusão: publicação antes de 2009 e sem apresentar cuidados no puerpério. Após a leitura e seleção, permaneceram apenas 10 artigos que falaram da relação do autocuidado materno e assistência da enfermagem, dos quais foi excluído 1 artigo por apresentar a temática voltada somente para puérpera adolescente, sendo um universo mais individual e restrito. Houve a validação da busca por dois pesquisadores convidados, que entendiam sobre a temática. $\mathrm{O}$ corpus da revisão foi composto por 9 artigos, e os dados foram organizados usando os programas da microsoft Word e Excel, além de serem construídos cinco quadros contendo: autor, ano de publicação, título, objetivo da publicação, local e ano da amostra, metodologia utilizada e o desfecho conclusivo da publicação.

\section{RESULTADOS}

No que diz respeito à caracterização dos estudos, nos anos de publicação dos artigos selecionados, observou-se que tiveram estudos realizados desde 2006 a 2015 e que foram publicados entre os anos de 2009 a 2017. Através dos descritores e pesquisa pelo tema, observou-se que a maioria dos artigos apresentaram temática voltada para um cuidado específico, como o cuidado na amamentação e com a puérpera adolescente. A pesquisa em língua estrangeira, trouxe poucos artigos falando do cuidado de enfermagem. A maioria dos artigos em inglês, falam do puerpério voltado apenas para os agravos de saúde e mortalidade materna.

\section{DISCUSSÃO}

Os resultados demonstram que ainda há um número considerável de mulheres com pouco conhecimento a respeito do seu autocuidado no período puerperal e o cuidado com o recém-nascido ${ }^{(12)}$. Pôde-se

\section{QUADRO 1 - Caracterização dos estudos relacionados aos cuidados maternos no período puerperal. Brasil, 2012-2017.}

\begin{tabular}{|c|c|c|c|c|c|}
\hline AUTOR, ANO & TÍTULO & OBJETIVO & $\begin{array}{c}\text { LOCAL, ANO, } \\
\text { AMOSTRA }\end{array}$ & MÉTODOS & DESFECHO \\
\hline $\begin{array}{l}\text { SILVA EC, } \\
\text { PEREIRA ES, } \\
\text { SANTOS WN } \\
\text { dos et al(8). } \\
2017\end{array}$ & $\begin{array}{l}\text { Puerpério e } \\
\text { assistência } \\
\text { de enfer- } \\
\text { magem per- } \\
\text { cepção das } \\
\text { mulheres. }\end{array}$ & $\begin{array}{l}\text { Conhecer a percepção } \\
\text { de mulheres sobre o } \\
\text { puerpério e assistência } \\
\text { de enfermagem. }\end{array}$ & $\begin{array}{l}\text { Realizado nas UBS do } \\
\text { Município de Ria- } \\
\text { chão- MA. Estudo com } \\
\text { mulheres entre } 20 \text { a } 40 \\
\text { anos. Pesquisa feita de } \\
\text { nov a dez } 2015 .\end{array}$ & Qualitativo & $\begin{array}{l}\text { Existem lacunas para melhor } \\
\text { qualidade de vida das puérperas } \\
\text { e a assistência de enfermagem } \\
\text { precisa ir além. }\end{array}$ \\
\hline $\begin{array}{l}\text { OLIVEIRA JFB, } \\
\text { QUIRINO GS, } \\
\text { RODRIGUES } \\
\text { DP }^{(9)} \cdot 2012\end{array}$ & $\begin{array}{l}\text { Percepção } \\
\text { das puérpe- } \\
\text { ras quanto } \\
\text { aos cuidados } \\
\text { prestados } \\
\text { pela equipe } \\
\text { de saúde no } \\
\text { Puerpério. }\end{array}$ & $\begin{array}{l}\text { Investigar a } \\
\text { experiência da } \\
\text { puérpera durante a } \\
\text { transição ao papel } \\
\text { materno a partir dos } \\
\text { cuidados dispensados } \\
\text { pela equipe de saúde. }\end{array}$ & $\begin{array}{l}\text { Realizado em } 4 \text { UBS do } \\
\text { município de Missão } \\
\text { Velha, Estado do Ceará } \\
\text { com } 14 \text { puérperas no } \\
\text { período de junho a } \\
\text { agosto de } 2007 \text {. }\end{array}$ & Qualitativo & $\begin{array}{l}\text { A assistência puerperal ainda } \\
\text { vem sendo negligenciada. As } \\
\text { orientações estiveram resumidos } \\
\text { à amamentação e aos cuidados } \\
\text { com o RN, omitindo a puérpera. }\end{array}$ \\
\hline $\begin{array}{l}\text { PEREIRA } \\
\text { MC, GRADIM } \\
\text { CVC(10). } 2014\end{array}$ & $\begin{array}{l}\text { Consulta } \\
\text { puerperal: } \\
\text { a visão do } \\
\text { enfermeiro e } \\
\text { da puérpera }\end{array}$ & $\begin{array}{l}\text { Avaliar a visão da } \\
\text { consulta puerperal sob } \\
\text { a perspectiva do enfer- } \\
\text { meiro, que a executa, e } \\
\text { da puérpera. }\end{array}$ & $\begin{array}{l}\text { Realizado com } 28 \\
\text { puérperas e } 10 \text { enfer- } \\
\text { meiras. Coletado em } 12 \\
\text { UBSF do município de } \\
\text { Alfenas-MG e ocorreu } \\
\text { no segundo semestre } \\
\text { de } 2012 \text {. }\end{array}$ & Qualitativo & $\begin{array}{l}\text { Os cuidados voltados à puérpera } \\
\text { estão deficientes. A dificuldade } \\
\text { para o enfermeiro pode levar a } \\
\text { perda de vínculo nessa fase de } \\
\text { pós-parto. }\end{array}$ \\
\hline
\end{tabular}


QUADRO 2 - Caracterização dos estudos relacionados aos cuidados maternos no período puerperal. Brasil, 2011-2012.

\begin{tabular}{|c|c|c|c|c|c|}
\hline AUTOR ANO & TÍTULO & OBJETIVO & LOCAL, ANO, AMOSTRA & MÉTODOS & DESFECHO \\
\hline $\begin{array}{l}\text { BERNAR- } \\
\text { DI, M.E; } \\
\text { CERRARO, } \\
\text { T.E; SEBOLD, } \\
\text { L.F.' } F^{(11)} .2011\end{array}$ & $\begin{array}{l}\text { Visita domiciliária } \\
\text { puerperal como } \\
\text { estratégia de cui- } \\
\text { dado de enferma- } \\
\text { gem na atenção } \\
\text { básica: revisão } \\
\text { integrativa. }\end{array}$ & $\begin{array}{l}\text { Realizar urna revi- } \\
\text { são integrativa de } \\
\text { literatura sobre o } \\
\text { conhecimento dos } \\
\text { cuidados de enferma- } \\
\text { gem no puerpério em } \\
\text { domicilio. }\end{array}$ & $\begin{array}{l}\text { Dados foram coletados } \\
\text { em periódicos nacionais } \\
\text { e internacionais BDENF, } \\
\text { SciELO, CINAHL, MEDLI- } \\
\text { NE e LILACS, no período } \\
\text { de janeiro de } 2006 \text { a } \\
\text { dezembro de } 2010 \text {. }\end{array}$ & $\begin{array}{l}\text { Revisão } \\
\text { integrativa da } \\
\text { literatura }\end{array}$ & $\begin{array}{l}\text { Pode-se concluir que } \\
\text { puérperas e enfermeiros } \\
\text { precisam aproximar-se } \\
\text { para que o cuidado seja } \\
\text { realmente efetivo, pauta-se } \\
\text { na reflexão e troca de } \\
\text { conhecimentos. }\end{array}$ \\
\hline $\begin{array}{l}\text { SILVA LR, } \\
\text { ARANTES } \\
\text { LAC, VILLAR } \\
\text { ASE, SANTOS } \\
\text { IMM et al }{ }^{(12)} \\
2012\end{array}$ & $\begin{array}{l}\text { Enfermagem } \\
\text { no puerpério: } \\
\text { detectando o } \\
\text { conhecimento das } \\
\text { puérperas para } \\
\text { o autocuidado e } \\
\text { cuidado com o } \\
\text { recém-nascido. }\end{array}$ & $\begin{array}{l}\text { Detectar o } \\
\text { conhecimento das } \\
\text { puérperas internadas } \\
\text { no alojamento } \\
\text { conjunto acerca do } \\
\text { seu autocuidado e } \\
\text { cuidado com o recém- } \\
\text { nascido (RN). }\end{array}$ & $\begin{array}{l}\text { Realizado com } 30 \\
\text { puérperas no alojamento } \\
\text { conjunto, participantes } \\
\text { das práticas educativas } \\
\text { da Maternidade do } \\
\text { HUGG/UNIRIO, em } 2009 .\end{array}$ & $\begin{array}{l}\text { Descritivo } \\
\text {-Exploratório }\end{array}$ & $\begin{array}{l}\text { Ainda há mulheres com } \\
\text { pouco conhecimento a } \\
\text { respeito do autocuidado } \\
\text { no período puerperal. Os } \\
\text { enfermeiros elaboraram } \\
\text { um material educativo } \\
\text { orientações para o cuidado } \\
\text { no domicílio. }\end{array}$ \\
\hline
\end{tabular}

QUADRO 3 - Caracterização dos estudos relacionados aos cuidados maternos no período puerperal. Brasil, 2013-2014.

\begin{tabular}{|c|c|c|c|c|c|}
\hline AUTOR ANO & TÍTULO & OBJETIVO & $\begin{array}{c}\text { LOCAL, ANO, } \\
\text { AMOSTRA }\end{array}$ & MÉTODOS & DESFECHO \\
\hline $\begin{array}{l}\text { RIBEIRO, } \\
\text { D.H.F; LU- } \\
\text { NARDI, V.L; } \\
\text { GOMES, G.C; } \\
\text { et al(13). } 2014\end{array}$ & $\begin{array}{l}\text { Vivências de } \\
\text { cuidado da } \\
\text { mulher: a voz das } \\
\text { puérperas. }\end{array}$ & $\begin{array}{l}\text { Conhecer como a } \\
\text { mulher vem viven- } \\
\text { ciando o seu cuidado } \\
\text { no puerpério. }\end{array}$ & $\begin{array}{l}\text { Realizado no primeiro } \\
\text { semestre de } 2008 \text {, em } \\
\text { um hospital do Sul no } \\
\text { Brasil, com dez puérpe- } \\
\text { ras egressas. }\end{array}$ & Qualitativo & $\begin{array}{l}\text { O cuidado familiar as puérperas } \\
\text { são influenciadas pelas tradi- } \\
\text { ções e pela cultura do seu meio. } \\
\text { A consulta de enfermagem } \\
\text { apresenta-se como um espaço } \\
\text { para a mulher tirar suas dúvidas, } \\
\text { aprender a cuidar e a ser cuidada. }\end{array}$ \\
\hline $\begin{array}{l}\text { MADALOZO, } \\
\text { F; XAVIER R. A. } \\
P^{(14)} .2013\end{array}$ & $\begin{array}{l}\text { Projeto consulta } \\
\text { puerperal de } \\
\text { enfermagem: } \\
\text { avaliando o } \\
\text { aprendizado } \\
\text { adquirido de } \\
\text { puérperas sobre } \\
\text { o pós-parto. }\end{array}$ & $\begin{array}{l}\text { Identificar o } \\
\text { aprendizado adquirido } \\
\text { das mulheres } \\
\text { sobre o pós-parto } \\
\text { atendidas pelo Projeto } \\
\text { Consulta Puerperal de } \\
\text { Enfermagem nos anos } \\
\text { de } 2009 \text { e } 2010 \text {. }\end{array}$ & $\begin{array}{l}\text { Realizado no Hospital } \\
\text { e Maternidade no } \\
\text { município de Ponta } \\
\text { Grossa, Paraná,Brasil. } \\
\text { Participaram do estudo } \\
177 \text { puérperas em } \\
\text { período pós-parto } \\
\text { mediato e tardio, nos } \\
\text { anos de } 2009 \text { e } 2010 \text {. }\end{array}$ & Quantitativo & $\begin{array}{l}\text { O estudo identificou o } \\
\text { aprendizado de puérperas em } \\
\text { média de } 87 \% \text { de respostas } \\
\text { certas, evidenciando a } \\
\text { importância da educação em } \\
\text { saúde no pós parto, visando a } \\
\text { autonomia no cuidado materno } \\
\text { e com o bebê. }\end{array}$ \\
\hline
\end{tabular}

QUADRO 4 - Caracterização dos estudos relacionados aos cuidados maternos no período puerperal. Portugal, 2015.

\begin{tabular}{|c|c|c|c|c|c|}
\hline AUTOR ANO & TÍTULO & OBJETIVO & LOCAL, ANO, AMOSTRA & MÉTODOS & DESFECHO \\
\hline $\begin{array}{l}\text { CORREIA, } \\
\text { T.I.G; PEREI- } \\
\text { RA,M.L.l.(15) } \\
2015\end{array}$ & $\begin{array}{l}\text { Os cuidados de } \\
\text { enfermagem e } \\
\text { a satisfação dos } \\
\text { consumidores } \\
\text { no puerpério }\end{array}$ & $\begin{array}{l}\text { Investigar a satisfação } \\
\text { das puérperas, em } \\
\text { internação, com os } \\
\text { cuidados especiali- } \\
\text { zado prestados pelos } \\
\text { enfermeiros num } \\
\text { serviço de obstetrícia } \\
\text { de uma unidade de } \\
\text { saúde de Portugal. }\end{array}$ & $\begin{array}{l}\text { A amostra foi constituída por } \\
\text { todas as puérperas inter- } \\
\text { nadas num serviço de obs- } \\
\text { tetrícia de uma unidade de } \\
\text { saúde do Norte de Portugal, } \\
\text { num total de } 120 \text { puérperas. } \\
\text { A coleta de dados foi através } \\
\text { de um questionário (entre o } \\
\text { mês de Fevereiro e o mês de } \\
\text { Abril de 2013). }\end{array}$ & $\begin{array}{l}\text { Transversal } \\
\text { analítico }\end{array}$ & $\begin{array}{l}\text { Revelaram que as mulheres } \\
\text { se encontravam muito satis- } \\
\text { feitas quanto aos cuidados } \\
\text { recebidos em geral ( } 50 \%) \text {, } \\
\text { da amamentação }(94 \%) \text { e } \\
\text { do autocuidado ( } 89 \%) \text {. Há } \\
\text { necessidade de maior eficácia } \\
\text { dos profissionais nos cuida- } \\
\text { dos prestados, para que as } \\
\text { puérperas se tornem mais } \\
\text { autônomas no autocuidado. }\end{array}$ \\
\hline
\end{tabular}




\section{QUADRO 5 - Caracterização dos estudos relacionados aos cuidados maternos no período puerperal. Uganda, 2006,} publicado em 2009.

\begin{tabular}{|c|c|c|c|c|c|}
\hline AUTOR ANO & TÍTULO & OBJETIVO & $\begin{array}{c}\text { LOCAL, ANO, } \\
\text { AMOSTRA }\end{array}$ & MÉTODOS & DESFECHO \\
\hline $\begin{array}{l}\text { PIUS OKONG, } \\
\text { J; BYAMU- } \\
\text { GISHA, F; } \\
\text { MIREMBE, R; } \\
\text { BYARUHANGA } \\
\text { E; STAFFAN } \\
\text { B (16) } \\
(2006)\end{array}$ & $\begin{array}{l}\text { Auditoria de mor- } \\
\text { bidade materna } \\
\text { grave no Uganda } \\
\text { - implicações para } \\
\text { a qualidade dos } \\
\text { cuidados obsté- } \\
\text { tricos }\end{array}$ & $\begin{array}{l}\text { O objetivo deste } \\
\text { estudo é explorar a } \\
\text { auditoria de morbida- } \\
\text { de obstétrica grave e o } \\
\text { conceito de near miss } \\
\text { (Incidente sem dano) } \\
\text { em quatro hospitais } \\
\text { de referência em } \\
\text { Uganda. }\end{array}$ & $\begin{array}{l}\text { A amostra foi cons- } \\
\text { tituída por puérperas } \\
\text { em quatro hospitais de } \\
\text { Uganda centro-leste do } \\
\text { continente africano. Os } \\
\text { dados foram extraídos } \\
\text { de registros clínicos } \\
\text { e de entrevistas com } \\
\text { pacientes, familiares e } \\
\text { profissionais de saúde. } \\
\text { (em 2004). }\end{array}$ & $\begin{array}{l}\text { Investigação } \\
\text { sistemática } \\
\text { exploratória }\end{array}$ & $\begin{array}{l}\text { Registros de } 685 \text { mulhe- } \\
\text { res foram examinados e } \\
229 \text { casos preencheram } \\
\text { os critérios. Hemorragia } \\
\text { obstétrica, ruptura do útero, } \\
\text { sepse puerperal e compli- } \\
\text { cações do aborto foram as } \\
\text { principais condições. Quase } \\
\text { metade dos casos estava } \\
\text { em casa quando os eventos } \\
\text { ocorreram. Mais da metade } \\
\text { dos casos demorou a procu- } \\
\text { rar atendimento. A auditoria } \\
\text { pode oferecer um estímulo } \\
\text { para melhorar a qualidade } \\
\text { dos cuidados obstétricos. }\end{array}$ \\
\hline
\end{tabular}

verificar que os cuidados voltados à puérpera estão deficientes, e os cuidados em relação ao sono e repouso, à higiene corporal, à sexualidade, à infecção puerperal e ao planejamento familiar são poucos relatados pelos diálogos presente neste estudo ${ }^{(8)}$.

Verificou-se o quanto a assistência puerperal desempenhada nos hospitais tem se mostrado limitada, pois grande parcela das puérperas afirmou não ter recebido nenhum tipo de orientação acerca dos cuidados nesta fase, alegando ainda haver sido seu período de internação exíguo, impossibilitando tal conduta ${ }^{(9)}$. Já nas que receberam orientações, pôde-se observar que os seus cuidados no puerpério estiveram resumidos apenas à amamentação e aos cuidados com o RN, omitindo atenção à grande protagonista desta fase, a puérpera ${ }^{(9)}$.

Uma das formas de amenizar a ocorrência dessas dificuldades e intercorrências suscetíveis a ocorrer no puerpério, é a potencialização das orientações no momento da alta hospitalar, pois é notável que as participantes do estudo se queixaram de intercorrências que aconteceram após a saída da maternidade ${ }^{(8)}$.

Observou-se a deficiência das orientações relacionadas ao cuidado integral

\section{Como profissionais}

\section{de saúde, temos}

que estar atentos,

planejar e realizar

ações com enfoque

na educação para

dar a possibilidade

à mulher de realizar

a continuidade

do autocuidado e

do cuidado com o

recém-nascido no

domicílio. à mulher que vivencia essa fase. Pôde-se constatar que o cuidado de enfermagem dispensado à puérpera durante o ciclo gravídico-puerperal não garante a autoconfiança necessária para o desempenho dos cuidados consigo mesma ${ }^{(10)}$.

Como profissionais de saúde, temos que estar atentos, planejar e realizar ações com enfoque na educação para dar a possibilidade à mulher de realizar a continuidade do autocuidado e do cuidado com o recém-nascido no domicílio ${ }^{(12)}$. O único trabalho que apresentou o projeto Consulta Puerperal de Enfermagem, desde 2006, atuou na educação em saúde da mulher, orientando-a quanto os principais assuntos do período puerperal ${ }^{(14)}$.

E outra pesquisa que elaborou um material educativo denominado Mãe \& Bebê - Pós-parto: orientações para o cuidado no domicílio contendo 52 páginas ilustradas e que foi financiado pela Agência de Cooperação Internacional do Japão (JICA), e que faz parte do acervo didático, do espaço educativo para trabalhar as práticas educativas com puérperas e seus recém-nascidos com alta hospitalar do alojamento conjunto do Hospital Universitário Gaffrée e Guinle (HUGG) ${ }^{(12)}$.

Algumas intercorrências ocorridas du- 
rante a fase puerperal, tanto com a mãe, quanto com o recém-nascido, podem ser decorrentes do despreparo profissional e/ou desinformação e até mesmo incompreensão das puérperas em relação às informações recebidas durante seu período pré-natal $^{(12)}$.

\section{CONCLUSÃO}

Pôde-se observar através das pesquisas, que as puérperas demonstram insegurança e desinformação quanto ao autocuidado e que as orientações realizadas pela enfermagem na alta hospitalar e visita puerperal, se dá principalmente atenção aos cuidados com recém-nascido, deixando uma lacuna no cuidado. Foi possível compreender a necessidade de existirem mais trabalhos sobre o autocuidado materno e incentivar os profissionais enfermeiros na orientação específica para puérpera.

\section{REFERÊNCIAS}

1. Corrêa MSM. Acolhimento no cuidado à saúde da mulher no puerpério. Cad. Saúde Pública. [internet] 2017 [cited 2019 nov 2] 33 (3). Available from: <https://www.scielosp.org/scielo. php?pid=S0102311X2017000305011\&script=sci_abstract.

2. Gomes GF, Santos APV dos. Assistência de enfermagem no puerpério. Revista Enfermagem Contemporânea. [internet] 2017 [cited 2019 nov 5] Outubrol;6(2):211-220. Available from: https://www5.bahiana.edu.br/index.php/enfermagem/article/ view/1407/1081.

3. Cassiano NA, Araújo MG, Holanda CSM, Costa RKS de. Percepção de enfermeiros sobre a humanização na assistência de enfermagem no puerpério imediato. J. res.: fundam. care. online [internet] 2015. [cited 2019 nov 5] jan./mar. 7(1):2051-2060. Available from: https://www.redalyc.org/ pdf/5057/505750945026.pdf.

4. Almeida MS, Silva IA. Necessidades de mulheres no puerpério imediato em uma maternidade pública de Salvador, Bahia, Brasil. Rev Esc Enferm USP. [internet] 2008. [cited 2019 nov 8] 42(2):347-54. Available from: http://dx.doi.org/10.1590/S008062342008000200019 .

5. Vieira SM, Bock LF, Zocche DA, Pessota CU. Percepção das puérperas sobre a assistência prestada pela equipe de saúde no pré-natal. Texto Contexto Enferm. [internet] 2011 [cited 2019 nov 9] 20(spe):25562. Available from: http://dx.doi.org/10.1590/ S0104-07072011000500032.

6. Giaretta DG, Fagundez F. Aspectos psicológicos no puerpério: revisão. Psicologia o portal dos periódicos. [internet] 2015 [cited 2019 nov 10]. Available from:https://www.psicologia.pt/artigos/ textos/A0922.pdf.

7. Dassoler MF, Ceretta LB, Soratto MT. Challenges Faced By Nurses In Puerperal Query. Revista interdisciplinar de estudos em saúde. [internet] 2017 [cited 2019 nov 10]. 6.n.2.p.162-176. Available from: http://periodicos.uniarp.edu.br/index.php/ries/ article/view/840/696.

8. Silva EC, Pereira ES, Santos WN dos, Silva RAR da, Lopes NC, Figueiredo TAM de, et al. Puerpério e assistência de enfermagem: percepção das mulheres. Rev enferm UFPE online. [internet] 2017 [cited 2019 nov 10] 11(Supl. 7):2826-33, jul., 2017. Available from: https://periodicos.ufpe.br/revistas/revistaenfermagem/article/download/11043/19180.

9. Oliveira JFB, Quirino GS, Rodrigues DP. Percepção das puérperas quanto aos cuidados prestados pela equipe de saúde no Puerpério. Revista da Rede de Enfermagem do Nordeste. [in- ternet] 2012 [cited 2019 nov 11] 3:1, pp. 74-84. Universidade Federal do Ceará, Fortaleza, Brasil. Available from: http://www. redalyc.org/articulo.oa?id=324027980010.

10. Pereira MC, Gradim CVC. Consulta puerperal: a visão do enfermeiro e da puérpera. Cienc Cuid Saude. [internet] 2014 [cited 2019 nov 12] Jan/Mar; 13(1):35-42. Alfenas, Minas Gerais, Brasil. Available from: http://periodicos.uem.br/ojs/index.php/CiencCuidSaude/article/view/19572/pdf_140.

11. Bernardi ME, Cerraro TE, Sebold LF. Visita domiciliária puerperal como estratégia de cuidado de enfermagem na atenção básica: revisão integrativa. Revista da Rede de Enfermagem do Nordeste. [internet] 2011 [cited 2019 nov 12] 12, pp. 10741080.Universidade Federal do Ceará, Fortaleza, Brasil. Available from: http://www.redalyc.org/articulo.oa?id=324027978025.

12. Silva LR, Arantes LAC, Villar ASE, Santos IMM, Guimarães EC da. Enfermagem no puerpério: detectando o conhecimento das puérperas para o autocuidado e cuidado com o recém-nascido. R. pesq: cuid. fundam. online [internet] 2012. [cited 2019 nov 13] abr./jun. 4(2):2327-37 Universidade Federal do Estado do Rio de Janeiro -UNIRIO. Available from: https://dialnet.unirioja. es/servlet/articulo?codigo=3971627.

13. Ribeiro DHF, Lunardi VL, Gomes GC, Xavier DM, Chagas MCS da. Vivências de cuidado da mulher: a voz das puérperas. Rev enferm UFPE on line. [internet] 2014 [cited 2019 nov 14] 8(4):820-6. Available from: https://periodicos.ufpe.br/revistas/ revistaenfermagem/article/download/9748/9861.

14. Madalozo F, Xavier RAP. Projeto consulta puerperal de enfermagem: avaliando o aprendizado adquirido de puérperas sobre o pós-parto. Revista Conexão UEPG. [internet] 2013 [cited 2019 nov 15] 9:1, pp. 154-161. Universidade Estadual de Ponta Grossa Ponta Grossa, Brasil. Available from: http://www.redalyc.org/ articulo.oa?id=514151729014.

15. Correia TIG, Pereira MLI. Os cuidados de enfermagem e a satisfação dos consumidores no puerpério. Nursing care and satisfaction of users in post-partum. Cuidados de enfermería y satisfacción de los consumidores en el puerpério. Rev. Eletr. Enf. [Internet]. 2015 [cited 2019 nov 15] jan./mar.;17(1):21-9. Available from: http://dx.doi.org/10.5216/ree.v17i1.28695.

16. Okong P, Amugisha JB, Mirembe F, Byaruhanga R, Bergstrom S. Audit of severe maternal morbidity in Uganda - implications for quality of obstetric care. Acta Obstetricia et Gynecologica Scandinavica. [Internet] 2006 [cited 2019 nov 15] 85: 7, 797804. Available from:https://www.tandfonline.com/doi/full/10.1 080/00016340600593331?scroll=top\&needAccess=true. 